\title{
An Automatic Registration of UV Images
}

\author{
Chuanying Wang \\ School of Electronic Science and Engineering \\ National University of Defense Technology \\ Changshan, China
}

\author{
Jixiang Sun \\ School of Electronic Science and Engineering \\ National University of Defense Technology \\ Changshan, China
}

\begin{abstract}
Corona discharge which occurs on high voltage equipment has being detected by Ultraviolet (UV) imaging technology for these years. Image processing technique is essential for $U V$ detection. Image registration technique is one of important methods for image processing. It is useful for further pattern segment of $\mathrm{UV}$ images and recognition study by image registration. In this paper, an image registration approach based on SURF (Speed Up Robust Features) is proposed. Firstly, the feature points were extracted using SURF; then the mapping relationship between images could be achieved using RSA(Random Sampling Algorithm ) and least square method; finally, transform parameter matrix could be established. The result of experiments shows that our method can achieve accuracy and practicability.
\end{abstract}

Keywords- UV image, image registration, SURF, RSA, least square method

\section{INTRODUCTION}

Corona discharge occurs on power equipment of which the insulation performance decreases under certain conditions. Corona discharge causes power loss, radio \& noise interferences and can accelerate degradation of various grid components and indicate a number of factors which may cause an outage or safety issue [1]. So it has important and necessary significance to inspect and detect the corona discharge in time. The information obtained during a UV inspection can help utilities detect the corona discharge and increase safety and reliability of the power system. Implementing this technology into a preventative maintenance program will save a considerable amount of money and manpower.

The wavelength of UV ray on which the power equipment radiates when the corona discharge occurs is $240 \sim 280 \mathrm{~nm}$. In this wavelength range, ozone layer in the atmosphere absorbs much of the light wave[2]. So, by detecting the UV ray of this wavelength range, UV imaging technology can inspect and judge the state of corona discharge.

The linchpin of UV imaging technology is how to extract the discharge region from UV images accurately and automatically. The discharge information of one single UV image is not adequate to extract the discharge region steadily. It is researched that the corona discharge points coruscate random in a region of equipment in UV images[3]. Considered this point, the information of the discharge region is needed to be swelled by a series of UV images. Because the UV detect-ion apparatus is usually installed on a helicopter the displacement of the discharge region in different images is changed. Thus, image registration is necessary to fix up the discharge region in the adjacent UV images.

\section{IMAGE REGISTRATION}

Current image registration techniques are generally divided into two broad categories: area-based and featurebased techniques. The feature-based methods which are more widely studied typically involve extracting features such as edge, contours, line intersections, corners, and then applying a match metric to find the correspondences between two images. Feature-based methods are significantly faster than area-based methods because of the reduction in the problem space.

An image registration algorithm suitable for UV images has to be accurate, automatic and reliable. It also should be able to handle all the occurring geometric distortion and grey level variations between the two images [4].

The purpose of this paper is to demonstrate that using a fast and accurate approach for control point extraction and using a novel method to satisfy all the constraints mentioned above and enhance the accuracy and speed of the registration. The proposed registration method is performed by using detector and descriptor of SURF[5]. Then, the efficient RSA method and least square method are used to find correct matching point pairs and achieve precise transform model.

\section{A. Control point extraction}

SURF is an algorithm which developed by Bay et al. to detect and describe local features in images. SURF is based on the scale-space representation of an image [6], and it is invariant to image scale and rotation and illumination, and to even affine transformation. The algorithm is flowed as follow:

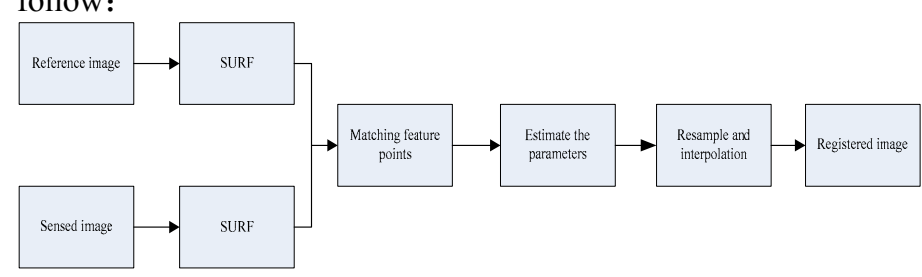

Figure 1. image registration algorithm based on SURF

\section{1) Fast-Hessian detector}


As we given a point $\hat{x}=(x, y)$ in an image $I$,the Hessian matrix at scale $\sigma$ is defined as

$$
H=\left(\begin{array}{ll}
L_{x x}(\hat{x}, \sigma) & L_{x y}(\hat{x}, \sigma) \\
L_{x y}(\hat{x}, \sigma) & L_{y y}(\hat{x}, \sigma)
\end{array}\right)
$$

Where $L_{x x}$ is the convolution of the Gaussian second order derivative $\frac{\partial^{2}}{\partial x^{2}} g(x, y, \sigma)$ with the image $I(x, y)$ and similarly for $L_{x y}$ and $L_{y y}$. The Gaussian second order derivative is defined as $g(x, y, \sigma)=\frac{1}{2 \pi \sigma^{2}} \mathrm{e}^{-\left(x^{2}+y^{2}\right) / 2 \sigma^{2}}$. In practice, the Gaussian needs to be discretized and cropped.

In order to accelerate the detection algorithm, the SURF detector pushes the approximation even further with box filters. These approximate second order Gaussian derivatives, and can be evaluated very fast using integral images, independently of size.

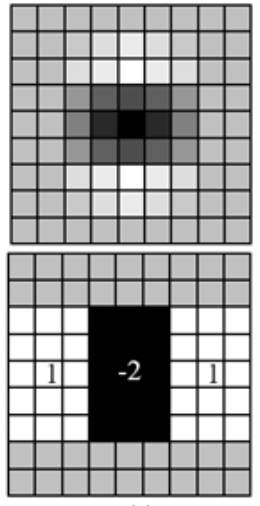

(a)

Figure 2.

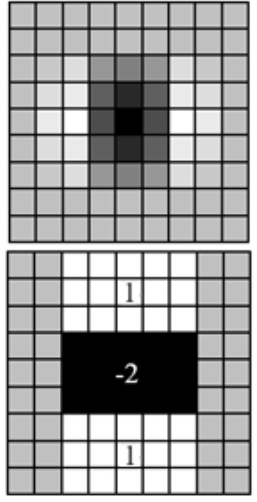

(b)

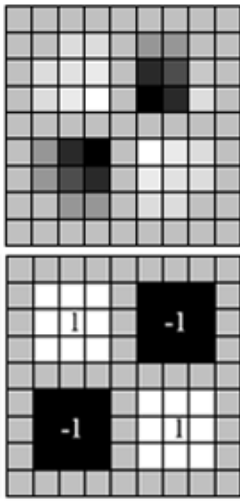

(c)
Upside: The Gaussian second order partial derivative;

Underside : the approximation for the second order Gaussian partial derivative.

Approximated Gaussian derivatives by box filter respectively, $D_{x x}, D_{x y}, D_{y y}$ are the convolution the image $I(x, y)$ with box filter. The approximated determinant of the Hessian which represents the blob response in the image can be expressed as:

$$
\operatorname{det} H=D_{x x} D_{y y}-\left(w D_{x y}\right)^{2}
$$

WhereEquation Chapter (Next) Section $1 w$ is the response of the filter [5].

Constructing scale pyramids of image, the images are repeatedly smoothed with a Gaussian and subsequently subsampled in order to achieve a higher level of the pyramid [6]. In order to localize interest points in the image and over scales, the non-maximum suppression in a $3 \times 3 \times 3$ neighborhood is applied. The maxima of the approximated determinant of the Hessian matrix are then interpolated in scale and image space with the method proposed by Brown et al. [7].So the characteristic scales and precise locations of the interest points can be simultaneously determined.

\section{2) Dominant orientation}

Firstly, in order to be invariant to rotation, the SURF descriptor identifies a dominant orientation for the interest points. We calculate the Haar-wavelet responses in $x$ and $y$ direction within the circular neighborhood of those interest points[8]. Then, the dominant orientation is estimated by calculating the sum of all Haar-wavelet responses within a sliding orientation window. The horizontal and vertical responses within the window are summed. The two summed responses then yield a new vector. The longest such vector lends its dominant orientation to the interest point.

\section{3) Extraction of the descriptor}

For the extraction of the descriptor, the first step consists of constructing a square region centered on the interest point and oriented along the orientation selected previously. The region is split up regularly into smaller $4 \times 4$ square subregions. For each sub-region, we compute Haar wavelet responses at $5 \times 5$ regularly spaced sample points. $d_{x}$ is the Haar wavelet response in horizontal direction and $d_{y}$ is the Haar wavelet response in vertical direction. Then, in order to enhance robustness of geometric transformation, the responses $d_{x}$ and $d_{y}$ and the absolute values of the responses $d_{x}$ and $d_{y}$ are summed up over each sub-region and form a first set of entries in the feature vector. Hence, each subregion has a 4D descryptor vector $V_{\text {sub }}$ for its underlying intensity structure i.e. $V_{\text {sub }}=\left(\sum d x, \sum d_{y}, \sum\left|d_{x}\right|, \sum\left|d_{y}\right|\right)$. For each interest point, it results in a descriptor vector of length $64(4 \times(4 \times 4))$. Finally, the descriptor vector should be turned into a unit vector.

\section{B. Matching}

After the descriptor is confirmed, we use one novel method--RSA and least square to simultaneously achieve correct correspondence and precise transform model. Supposing that $N_{1}$ is the total number of all control points of sensed image $I_{1}$ and $N_{2}$ is the total number of all control points of reference image $I_{2}$. For either control point $n_{1 i}$ in set $N_{1}, n_{2 j}$ and $n_{2 j}^{\prime}$ are the minimum Euclidean distance between the control points and $n_{1 i}$. The corresponding Euclidean distance is $d_{i j}$ and $d_{i j}^{\prime}$. If $d_{i j} \leq \alpha d_{i j}^{\prime}, n_{1 i}$ and $n_{2 j}$ are considered a corresponding matching pair. After all sensed image control points have been computed, we can find all latent matching pairs in sensed image and reference image.

\section{Parameter estimation}

After the all matching pairs achieved, parameter matrices should be computed between sensed image and reference 
image. Supposing the affine transformation parameter matrices are defined as

$$
\begin{aligned}
& M=\left(\begin{array}{ccc}
m_{1} & m_{2} & m_{3} \\
m_{4} & m_{5} & m_{6} \\
0 & 0 & 1
\end{array}\right) \\
& \text { If } p=\left(x_{1}, y_{1}\right) \text { and } q=\left(x_{2}, y_{2}\right) \text { is a matching pair, it can }
\end{aligned}
$$

be found that

$$
\left(x_{1}, y_{1}, 1\right)^{\mathrm{T}}=\left(\begin{array}{ccc}
m_{1} & m_{2} & m_{3} \\
m_{4} & m_{5} & m_{6} \\
0 & 0 & 1
\end{array}\right)\left(x_{2}, y_{2}, 1\right)^{\mathrm{T}}
$$

For discarding the wrong matching pairs, we use three random points matching pairs to compute the matrix $M$ Then; we can choose those point pairs whose distance of space localization less than certain threshold as the possible correct matching point pairs. The error should be minimized by least square method, and the matrix $M$ should be recomputed by the correct matching point pairs after that. Thereby, final precise transform matrix can be established.

\section{EXPERIMENTAL RESULTS}

To evaluate the accuracy and speed of the proposed method which will be compared with the method based on SIFT and BBF [7], we use the sensed image being the affine transformed and the reference one being illumination changed (see Fig. 3). The size of the reference image is $720 \times 576$ pixels and the sensed image is $266 \times 278$ pixels.

We use the proposed method that control points are extracted based on SURF and correct matching point pairs and achieve precise transform model are found based on RSA method and least square method. The implementation of the algorithms are done in Matlab2009 on a standard PC Intel(R) Pentium(R) $4 \mathrm{CPU}(2.8 \mathrm{GHz})$ with $1 \mathrm{G}$ memory.

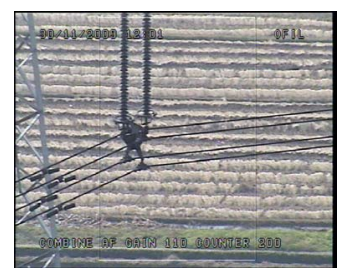

(a)

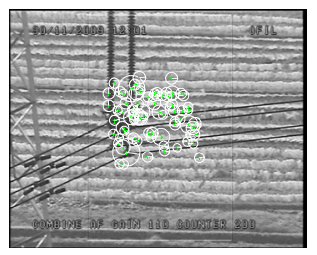

(c)

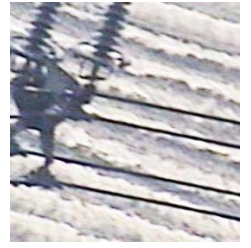

(b)

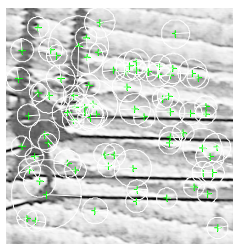

(d)

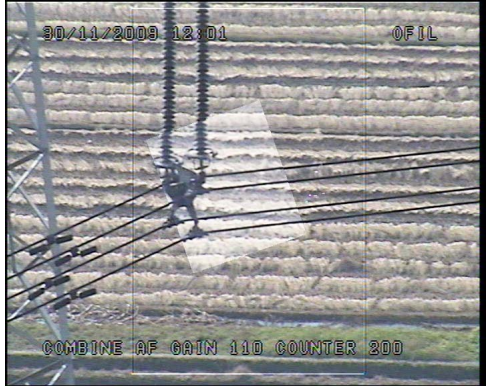

(e)

Figure 3. Registration of a pair of test images. (a) Reference image.(b) Sensed image. (c) The set of final matched control points (green crosses) in Image (a). The wh-ite circle's radius and the lines in the circle represent the character scales and the dominant orientation of the SURF points

respectively. (d) The corresponding set of final matched control points in Image (b). (e) The registration result.

The time used the method proposed above is 5.1734 seconds and that used the method which is compared with is 370.7581 seconds. It is note that our proposed registration algorithm is obviously faster than the method based on SIFT.

\section{SUMMARIES}

As a newly emerging technology, UV detection is welcome by power system-m and it will be valuable for deeply research and spread in the future. The difficulty of UV detection is how to extract the discharge region. In order to solve the problem by swelling the information of UV images accurately and automatically, we study an effective UV images registration. The experimental results show the proposed method satisfies the demand of UV image registration. It set the stage for the further extracting the discharge region.

However, because the UV detection apparatus is usually installed on a helicopter, some UV images recorded by the UV apparatus may be indistinct, which needs us to carry on the further research.

\section{REFERENCES}

[1] Mazen Abdel-Salam.Corona power loss determination on multi-phase power transmission lines[J],Electric Power Systems Research 58(2), 2001,PP:123-132

[2] Y.P.LIU;H.B.Wang .Test study on corona onset voltage of UHV transmission lines based on UV detection[C], International Conference on High Voltage Engineering and Application,2008 ,PP:387-388.

[3] Bin Ma;Wenjun Zhou.Corona discharge of the severe non-uniform electric field based on the UV-light imaging technology[J], High Voltage Engineering32(7), 2006,PP:13-15

[4] B.Zitova;J. Flusser. Image registration methods: a survey[J], Image and Vision Computing 21 (11), 2003,977-1000.

[5] H.Bay; A.Ess; T.Tuytelaars; L.Van Gool. Speeded-Up Robust Features (SURF) $[\mathrm{J}]$, Computer Vision and Image Understanding 110(3), 2008,PP:346-359.

[6] T. Lindeberg. Scale-space for discrete signals[J], IEEE Transaction on Pattern Analysis and Machine Intelligence 12(3), 1990,PP:234254 . 
[7] D.G.Lowe. Distinctive image features from scale-invariant keypoints[J], the International Journal of Computer Vision 60(2), 2004, PP:91-110.
[8] M.Brown; D.Lowe. Invariant features from interest point groups[J], Proc BMVC, 2002,PP: 656-665.

Published by Atlantis Press, Paris, France.

(C) the authors 